\title{
Fibrolamellar hepatocellular carcinoma presenting as a gall bladder mass
}

\author{
Popli $\mathrm{MB}^{1 *}$ and Popli $\mathrm{V}^{2}$ \\ ${ }^{1}$ Department of Radiological Imaging, Institute of Nuclear Medicine and Allied Sciences (INMAS), Lucknow Road, Delhi, India \\ ${ }^{2}$ Department of Pediatrics, BSA hospital, Rohini, Delhi, India
}

\begin{abstract}
Fibrolamellar hepatocellular carcinoma (FL-HCC) is an uncommon histological subtype of Hepatocellular carcinoma, which seldom invades the gall-bladder (GB). Here we report a case of FL-HCC invading the GB, that resembled gall bladder carcinoma invading the liver on both US and CT. A central calcification and/or central stellate scar that are clue to the diagnosis of FL-HCC were absent.
\end{abstract}

\section{Case Report}

A 20-year-old male was referred for radiological evaluation, with complaint of continuous pain right hypochondrium of one-month duration. The pain was of moderate to severe intensity, not radiating to back or shoulders. No other symptoms were present. There was no history of alcohol abuse or previous hepatitis. On physical examination, an ill defined compressible tender mass was palpated below the right costal margin in the mid-clavicular line, moving with respiration. Routine blood investigations and liver function tests were within normal limits.

Ultrasound of the patient revealed a mass of mixed echogenicity arising from the anterior wall of the gall bladder (GB) and invading the liver (Figure 1). Another mass predominantly hypoechoic with non-homogenous internal echoes was found in proximity to the first mass, in right lobe of liver. Computed tomography showed a polypoidal

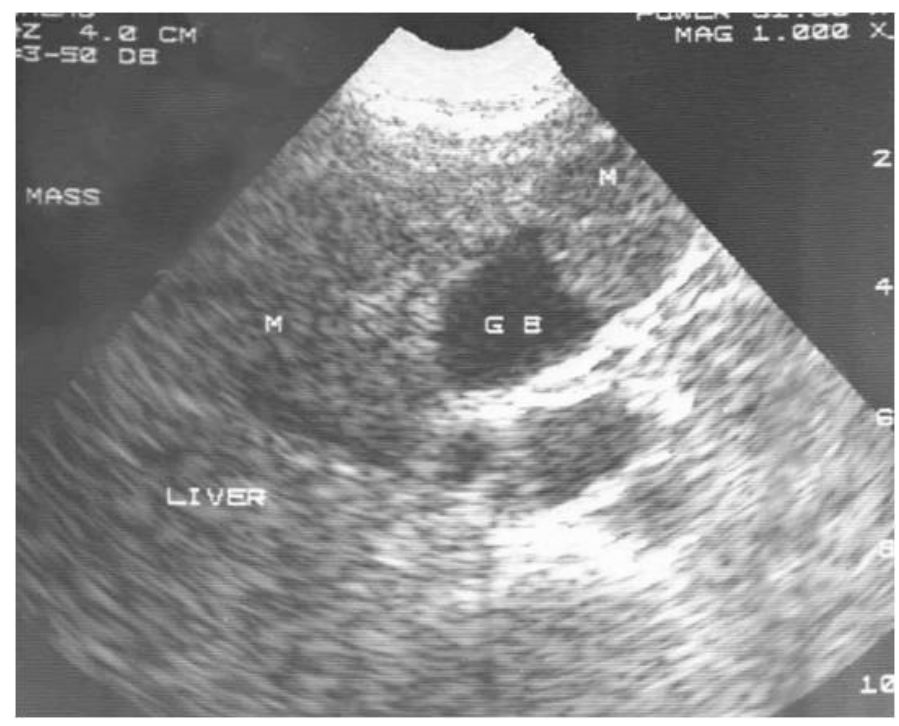

Figure 1. US Picture showing GB mass invading the Liver. Another mass is seen in right lobe of Liver (GB- Gall bladder; M- mass) mass arising from the anterior wall of the GB and protruding into the lumen (Figure 2). Mass was also extending into the adjacent area of the liver. On the right side of the GB, another focal mass of variegated appearance was seen. A probable diagnosis of carcinoma of the gallbladder with a secondary in liver was suggested.

Fine needle aspiration cytology was performed under ultrasound guidance. Cytological examination from the gall-bladder mass and the liver mass, were of similar morphology. The highly cellular smears were composed of tumor cells resembling normal liver but were at least 3-4 times larger. The cells were arranged in trabeculae, loosely cohesive groups and lying singly. Parallel rows of spindle shaped fibrocytes were seen intermingled with the neoplastic cells. The cells had a large nucleus with prominent nucleoli. Cytoplasmic bile pigments were seen in many cells. Some of the nuclei showed intra nuclear cytoplasmic inclusion. Few atypical mitotic figures were also seen (Figure 3). A probable diagnosis of FL-HCC was given.

Patient subsequently underwent right extended hepatectomy. Histopathological examination of the resected mass showed a circumscribed, bile stained, tumor of $7 \times 5 \mathrm{~cm}$ in right lobe near gall bladder fossa. It extended to the capsule focally and also up to the resected margin of the liver. The tumor was infiltrating through the wall of the gall bladder at the fundus as a polypoidal mass. Sections showed features of a hepato- cellular carcinoma, Fibrolamellar type.

FL-HCC is a distinct clinico-pathological entity [1-2]. It is a separate from the usual HCC which is associated with cirrhosis or hepatitis, frequently is non-resectable on diagnosis, and has an overall poor prognosis $[3,4]$. FL-HCC tends to occur in the young $[5,6]$. The reported range is 5-35 years, with a mean of 23 years and without

${ }^{\star}$ Correspondence to: Popli MB, Department of Radiological Imaging, INMAS, Delhi, India, Tel :011-23905285; Fax: 011-23930396; E-mail: manju_popli@rediffmail.com

Key words: liver, fibrolamellar, hepatocellular carcinoma

Received: January 03, 2019; Accepted: January 11, 2019; Published: January 14, 2019 


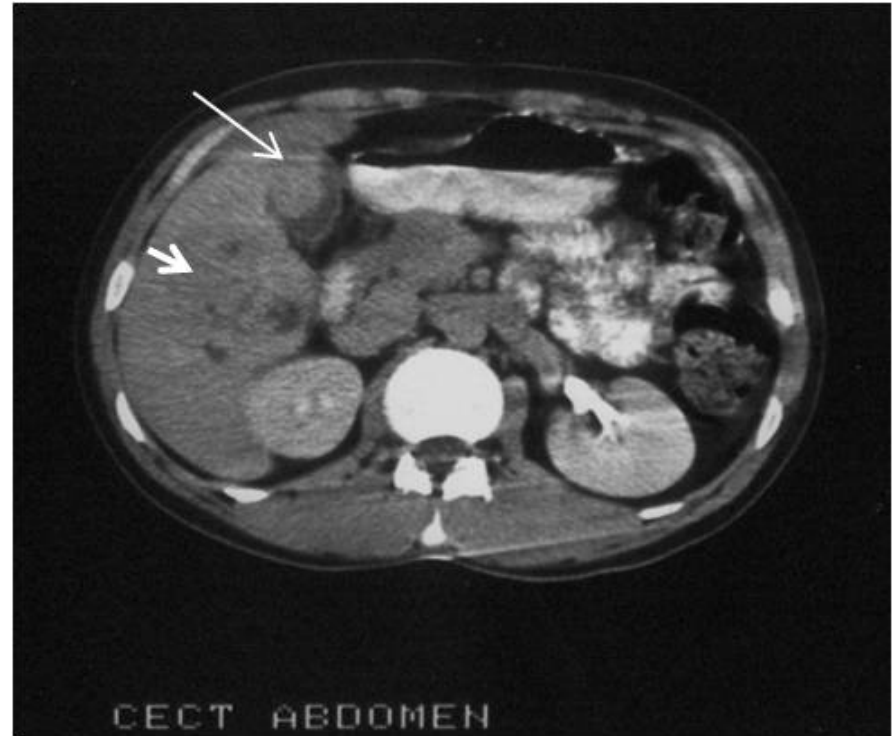

Figure 2. CT picture showing a polypoidal mass arising from anterior wall of GB and protruding into the lumen (long arrow). Another focal mass of variegated appearance also seen (short arrow)

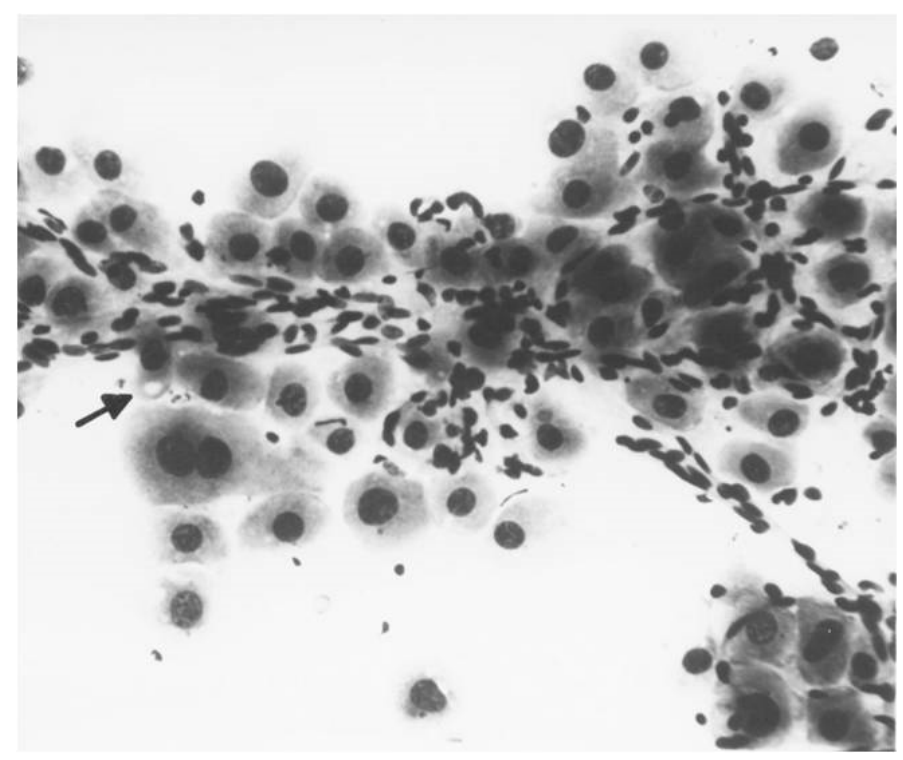

Figure 3. FNAC of the liver mass showing parallel rows of fibrocytes intermingled with tumor cells. Well defined pale body seen (arrow) in the cytoplasm of some cells. (X 200)

any sex predilection. It also lacks specific association with cirrhosis, hepatitis B virus infection, use of oral contraceptives and alcohol abuse, all of which are implicated in HCC $[7,8]$. Many studies have provided the evidence of better prognosis of FL-HCC compared to usual HCC: 32 months versus 6 months average survival, $48 \%$ versus $17 \%$ resectability, and $63 \%$ versus $30 \% 5$ years survival after successful resection [8].

Hepatocellular carcinoma has three growth patterns: a solitary lesion, multifocal lesions or diffuse hepatic infiltration. Larger masses tend to be heterogeneous and to have a central area of necrosis and abnormal internal vessels. On sonography, hepatocellular carcinoma has a variable appearance. FL-HCC appears as well-defined mass of variable echogenicity on ultrasound [9]. In our case it was a welldefined mass protruding into the GB. On CT imaging FL-HCC can be well defined or ill defined. Calcification is seen in $40-68 \%$ cases. Central stellate scar is seen in $65-70 \%$ cases. Radiating fibrotic bands and septae are also seen. Presence of calcification in the central scar is a diagnostic feature of FL- HCC [10]. None of these were identifiable in our case.

FL-HCC is a rare variant of HCC and is poorly understood. Young people or children are often the target individuals with this type of cancer. It is important to differentiate FL-HCC from HCC for which resection is often not the treatment of choice [11,12]. imaging and clinical correlation helps to identify FL- HCC in most cases but biopsy is required to confirm the definitive diagnosis. Distinguishing it from HCC avoids misdiagnosing and mistreating a potentially curable neoplasm.

\section{Conflict of interest}

Nil

\section{Funding}

Nil

\section{References}

1. Michael Torbenson (2012) "Fibrolamellar Carcinoma: 2012 Update," Scientifica 743790: 15 .

2. Liu S, Chan KW, Wang B, Qiao L (2009) Fibrolamellar hepatocellular carcinoma. Am $J$ Gastroenterol 104: 2617-24.

3. Fernandez MP, Redvanly RD (1998) Primary hepatic malignant neoplasms. Radiol Clin North Am 36: 333-348. [Crossref]

4. Schafer DF, Sorrell MF (1999) Hepatocellular carcinoma. Lancet 353: 1253-1257. [Crossref]

5. el-Serag HB (2001) Epidemiology of hepatocellular carcinoma. Clin Liver Dis 5: 87107. [Crossref]

6. Larcos G, Sorokopud H, Berry G, Farrell GC (1998) Sonographic screening for hepatocellular carcinoma in patients with chronic hepatitis or cirrhosis: an evaluation. AJR Am J Roentgenol 171: 433-435. [Crossref]

7. Herman P, Chagas AL, Perini MV, Coelho FF, Fonseca GM, et al. (2014) Surgical treatment of fibrolamellar hepatocellular carcinoma: an underestimated malignant tumor? Hepatobiliary Pancreat Dis Int 13: 618-621. [Crossref]

8. El-Serag HB, Davila JA (2004) Is fibrolamellar carcinoma different from hepatocellular carcinoma? A US population-based study. Hepatology 39: 798-803. [Crossref]

9. Kelvin HYL, O’Malley ME, John RK, Haider M, Hanbidge A (2003) Hepatocellular Carcinoma: Imaging and imaging- guided intervention. AJR 180: 1015-1022.

10. Ganeshan D, Szklaruk J, Kundra V, Kaseb A, Rashid A, et al. (2014) Imaging features of fibrolamellar hepatocellular carcinoma. AJR Am J Roentgenol 202: 544-552. [Crossref]

11. Vishnu N, Kulkarni AV, Vidhyalakshmi S, Sambandam S, Garg P, et al. (2017) Fibrolamellar variant of hepatocellular carcinoma presenting during pregnancy: management dilemmas. Ann Hepatobiliary Pancreat Surg 21: 48-51. [Crossref]

12. Stipa F, Yoon SS, Liau KH, Fong Y, Jarnagin WR, et al. (2006) Outcome of patients with fibrolamellar hepatocellular carcinoma. Cancer 106: 1331-1338. [Crossref]

Copyright: (C2019 Popli MB. This is an open-access article distributed under the terms of the Creative Commons Attribution License, which permits unrestricted use, distribution, and reproduction in any medium, provided the original author and source are credited. 